\title{
A profile of OR research and practice published in the Journal of the Operational Research Society
}

\author{
$\mathrm{K}_{\text {Katsaliaki }}{ }^{1 *}, \mathrm{~N}_{\text {Mustafee }}{ }^{2}$ and YK Dwivedi ${ }^{2}$ \\ ${ }^{1}$ International Hellenic University, Thermi, Greece; and ${ }^{2}$ Swansea University, Swansea, UK \\ Journal of the Operational Research Society (2011) 62, 1596-1597. doi:10.1057/jors.2010.183
}

Correction to: Journal of the Operational Research Society (2011) 61, 82-94, doi:10.1057/jors.2009.137

The authors would like to thank Professor Mingers for highlighting errors in our paper and apologise for the inadvertent mistakes that led to any missing or incorrect information being presented. The following amendments and additions address the points raised.

(1) In Table 7 of Katsaliaki et al (2010) the affiliation of the most prolific authors, who have had multiple affiliations over the past decade, has been defined by the affiliation noted in the majority of the articles published by the authors in question over the examined period. In the case of Professor Mingers we 'incorrectly' noted the University of Warwick as his affiliation, since six out of a total of nine publications attributed to him during this period were written under the University of Kent affiliation (and only the remaining three articles were written under the University of Warwick affiliation). The affiliation of Professor Mingers in Table 7 should be corrected to read University of Kent. It is also noted that the results of Table 5, which present a list of the top 20 universities that published in JORS between 2000-2009, are not affected as the University-specific statistics are based on the authors' affiliation stated in each article.

(2) The comment following Table 7 should be corrected and the name of Professor Mingers should appear there together with Professor Ormerod and Professor Robinson. It should read: 'On the other hand, the largest number of first-authored articles were contributed to by Berman (eight articles) and Podinovski (seven articles), followed by Mingers, Ormerod and Robinson, who are the first authors with six articles'.

\footnotetext{
${ }^{*}$ Correspondence: $K$ Katsaliaki, School of Economics and Business Administration, International Hellenic University, 14th Klm ThessalonikiN.Moudania, Thessaloniki, Thermi 57001, Greece.
}

(3) The comment following Table 8 also needs correction. It should read: 'Nevertheless, the first three most popular articles remain the same in the two tables, both in terms of total and average citations. On the other hand, from the 13 remaining articles only four are common between Tables 8 and 9. These articles are Teng (2002), Mingers (2000), Ulrich (2003) and Mosheiov (2001)'.

(4) In the discussion part of our paper the comparison is clearly made between the most productive (in terms of number of publications) authors which appear in Table 1 of Ranyard's paper and Table 7 in our paper. In these comparable lists only Laporte appears in both and not Mingers. In Katsaliaki et al (2010) no data is presented for the most cited authors and therefore no comparison is being made with Table 3 of Ranyard's paper. However, it is well worth making a comment that 'Mingers is presented as one of the most cited JORS authors over the 80 s and 90 s and also as one of the most prolific authors from 2000 to 2009.' In the same manner we could also mention that 'Drezner and Gupta appear as the authors of some of the most cited $J O R S$ papers during the 80 s and 90 s and also in the list of the most productive authors during 2000-2009'.

The authors would like to stress that although these are important details, they do not change the overall messages of the paper.

Finally, the authors would like to take this opportunity to clarify the data presented in Table 5; we note that this clarification is not related to the issues raised by Professor Mingers. Table 5 lists the top 20 universities that published in JORS between 2000-2009. In this table in columns 1 and 2 HEC Montreal is listed as the 19th most prolific institution with a total of 13 JORS publications. However, HEC Montreal was formerly known (before 2002) as École des Hautes Études commerciales de Montréal. Nevertheless, as the authors were not aware of the change in the name of the institution, they treated HEC Montreal and École des 
Hautes Études commerciales de Montréal as two different institutes resulting in HEC Montreal obtaining a lower position than it would have got otherwise. In addition, two of the authors mainly associated with HEC Montreal, namely Laporte $\mathrm{G}$ and Cordeau JF, in some of their publications appear with two affiliations; the second being HEC Montreal or École des Hautes Études commerciales de Montréal. In these cases the database counted only the first affiliation as the representative one. Combining HEC Montreal and École des Hautes Études commerciales de Montréal as one entity (as it should have been the case from the beginning) and defining HEC Montreal as the main affiliation of the two authors in the articles where they appear with two affiliations, places HEC Montreal: (i) in columns 1 and 2 in the 9th position with 21 papers; (ii) in columns 3 and 4 in the 15th position (new entry) together with Hanyang University, University of Hong Kong Polytechnic, Middle East Technical University and
University of Sheffield with 15 authors; and finally (iii) in columns 5 and 6 HEC Montreal is positioned 7th with 40 total contributions. However, this may not be treated as an error on the part of the authors, since it would have be very time consuming to methodologically search through the names of the vast number of entities (universities, research centres, commercial organisations, etc with which the authors have affiliation) in order to identify a change in name of an organisation.

\section{References}

Katsaliaki K, Mustafee N, Dwivedi Y, Williams T and Wilson J (2010). A profile of OR research and practice published in the Journal of the Operational Research Society. J Opl Res Soc 61: 82-94.

Ranyard J (2001). Editorial. J Opl Res Soc 52: 1-3. 